\title{
DIAGNÓSTICO Y MANEJO DE PACIENTES CON SÍNDROME DE VEJIGA HIPERACTIVA EN LAS CONSULTAS DE UROLOGÍA Y ATENCIÓN PRIMARIA EN ESPAÑA.
}

\author{
Mํ Justa García Matres y Francisco José Brenes Bermúdez'.
}

Servicio de Urología. Hospital Universitario La Paz. Madrid. Medicina de familia'. Centro de Salud Llefià. Badalona. España.

\begin{abstract}
Resumen.- OBJETIVO: El Síndrome de Vejiga Hiperactiva $(\mathrm{SVH})$, a pesar de presentar una elevada prevalencia y un impacto importante en la vida del paciente, es un trastorno mal conocido y poco abordado en la práctica asistencial. El objetivo del estudio es conocer las diferentes técnicas y procedimientos empleados en el diagnóstico de SVH, tanto por urólogos como por médicos de atención primaria (AP), en condiciones de práctica clínica habitual en España.
\end{abstract}

MÉTODOS: Se administró un cuestionario estandarizado a 748 médicos especialistas en urología y 696 médicos de AP. Dicho cuestionario recogía información sobre el manejo de los pacientes que acudían a su consulta con síntomas urinarios sugestivos de SVH.

RESULTADOS: Un 64,8\% de los urólogos afirmaron seguir algún protocolo estandarizado para el diagnóstico de pacientes con SVH. Un 58,4\% de los médicos de
AP afirmaron no derivar a los pacientes con síntomas urinarios sugestivos de SVH. Un 77,8\% de los urólogos afirmaron que los pacientes que venían derivados desde AP llegaban sin ningún diagnóstico ni estudio previo. En ambas especialidades la historia clínica, la exploración física y el análisis de orina son procedimientos que forman parte de la práctica diagnóstica habitual. Otras medidas como son el diario miccional y los cuestionarios de síntomas o de evaluación de la calidad de vida son aún poco utilizados en el diagnóstico y manejo del $\mathrm{SVH}$.

CONCLUSIÓN: Si bien el SVH es una condición que conlleva una gran afectación de la Calidad de Vida (CdV) y de las actividades diarias de los pacientes que lo padecen, todavía está poco abordada desde el punto de vista asistencial. Es importante establecer estrategias de detección de los síntomas en la práctica clínica.

Palabras clave: Síndrome de vejiga hiperactiva. Diagnóstico. Manejo clínico. Atención primaria. Urología.

Summary.- OBJECTIVES: Despite its high prevalence and its important impact on patient's life, overactive bladder is a disorder poorly known and not usually tackled in daily clinical practice. The aim of this study is to know the main procedures and techniques used by both urologists and general practitioners to diagnose overactive bladder in usual clinical practice in Spain.

METHODS: A standardized questionnaire was administered to 748 physicians specialized in urology and to 696 primary care physicians or general practitioners. The questionnaire collected information about the management of patients who attended surgery with urinary symptoms suggesting overactive bladder. 
RESULTS: A total of $64.8 \%$ of urologists argued they followed a standardized protocol in order to diagnose patients suffering from overactive bladder. Concerning primary care physicians, $58.4 \%$ of them admitted not referring patients with urinary symptoms suggesting overactive bladder to specialist. Up to $77.8 \%$ of urologists confirmed that referred patients from primary care had no previous exam or diagnosis. Health history, physical examination and urinalysis were the usual diagnosis procedures in clinical practice conditions for both specialities. Other instruments such as the micturition diary or the questionnaires to assess symptoms or patients' quality of life are still little used for the diagnosis and management of overactive bladder.

CONCLUSIONS: Although overactive bladder is a condition that causes a high impairment in the quality of life (QoL) and daily activities of those patients suffering from it, it still remains poorly tackled by doctors. Therefore, it is important to define strategies to detect its symptoms in the clinical practice.

Keywords: Overactive bladder. Diagnosis. Clinical management. Primary care. Urology.

\section{Financiado con la ayuda de Astellas Pharma, S.A.}

\section{INTRODUCCIÓN}

El Síndrome de Vejiga Hiperactiva (SVH) se definió en el año 2002 por la International Continence Society (ICS) como: urgencia, con o sin incontinencia de urgencia, generalmente acompañada de frecuencia y nocturia en ausencia de infección probada $u$ otra enfermedad demostrable (1). Aunque la etiología del SVH es desconocida se sospecha una etiología multifactorial, sugiriendo como posibles causas las alteraciones nerviosas centrales y periféricas o las alteraciones de la musculatura propia de la vejiga urinaria (2).

La prevalencia del SVH es elevada y aumenta con la edad. En un estudio epidemiológico europeo realizado en el 2000 se observó que la prevalencia total del $\mathrm{SVH}$ oscilaba entre el $12 \%$ y el $22 \%$ en personas de 40 o más años de edad (3). En EEUU el estudio NOBLE demostró una prevalencia del $16 \%$ en personas mayores de 17 años (4). En un estudio epidemiológico realizado en España en el año 2004, se registró una prevalencia de SVH en personas de edad igual o superior a los 40 años del $21,5 \%$ (5).

A pesar de la elevada prevalencia de $\mathrm{SVH}$ y de su impacto en la calidad de vida del pacien- te, se ha observado que un porcentaje importante de pacientes afectados (entre un 50 y un $80 \%$ ) no consultan por este motivo (6-8). Según un estudio realizado en el ámbito de la $A P$, entre los principales motivos de no consulta argumentados por los pacientes destacaba el hecho de que éstos consideraban que el problema era poco o nada importante y que era una situación normal asociada al envejecimiento para la que no existe una solución posible (9). Por otro lado, este mismo estudio identificó que en un elevado porcentaje de pacientes que solicitaban ayuda al médico de AP, éste no adoptaba ninguna actitud diagnóstica ni terapéutica. Todo ello lleva a pensar en que existe un infradiagnóstico e infratratamiento del problema (6, 10-15). Este hecho es especialmente importante en las fases más leves. El SVH es en realidad un trastorno mal conocido y poco abordado tanto desde la AP como desde especialidades como la urología $(10,16)$.

Con objeto de aumentar la calidad asistencial en este tipo de pacientes, es fundamental establecer una estrategia de detección de los síntomas en la práctica clínica de los médicos de AP y de los especialistas, así como poner en marcha procesos de diagnóstico siguiendo protocolos de referencia según los recursos y el nivel asistencial (10). En el año 1999 el Grupo de trabajo de Salud Vesical de la Asociación Española de Urología (AEU) (17) estableció las líneas generales de actuación para el diagnóstico de la incontinencia urinaria. A nivel internacional, la European Association of Urology (EAU) publicó en 1999 unas recomendaciones sobre la Incontinencia Urinaria (18), mientras que la International Consultation on Incontinence (ICl) publicó, en el año 2002, las recomendaciones sobre la Evaluación y Tratamiento de la Incontinencia Urinaria (19). Todas ellas tienen en común la diferenciación entre una evaluación inicial de la patología mediante una serie de herramientas y una evaluación especializada que pasaría por un estudio urodinámico, especialmente requerido tras un fracaso terapéutico o quirúrgico o ante la sospecha de neuropatía.

El objetivo principal de este estudio es conocer las diferentes técnicas y procedimientos empleados en el diagnóstico de SVH, tanto por médicos de AP como por urólogos, en condiciones de práctica clínica habitual en España.

\section{MATERIAL Y MÉTODOS}

Se diseñó un cuestionario estandarizado que fue administrado a una muestra de 748 médicos especialistas en urología y 696 médicos de AP. La recogida de datos se realizó entre julio y diciembre de 2005. 
La encuesta administrada a los médicos de ambas especialidades recogía información sobre el manejo de los pacientes que acudían a su consulta con síntomas urinarios sugestivos de SVH. El cuestionario final incluyó 15 preguntas para los urólogos y 13 para los médicos de AP divididas en 2 secciones comunes a cada especialidad: 1) perfil de derivación de los pacientes tratados y 2) técnicas y pruebas diagnósticas utilizadas, incluyendo el uso de diario miccional y de cuestionarios estandarizados para la identificación de los síntomas y la evaluación de la calidad de vida. En el caso de los médicos de AP se incluyó una sección adicional sobre la actualización de la información sobre la patología urinaria. En el caso de los urólogos se incluyó también una sección adicional que hacía referencia a la utilización de protocolos de actuación en condiciones de práctica clínica habitual.

Se realizó un análisis descriptivo de las respuestas obtenidas por los investigadores participantes en el estudio estratificando según la especialidad del médico. Para la descripción de variables continuas se utilizaron las medidas de tendencia central. Las variables categóricas nominales se resumieron utilizando el recuento de casos por cada categoría y la frecuencia relativa al total de respuestas. Se comparó el manejo de los pacientes con SVH según la especialidad del médico, utilizando la prueba de la ji cuadrado en el caso de variables categóricas y la prueba de la t de Student en el caso de variables continuas.

El análisis de datos se realizó empleando el paquete estadístico SPSS 10.1. En todas las pruebas estadísticas se consideró un nivel de significación estadística de 0,05.

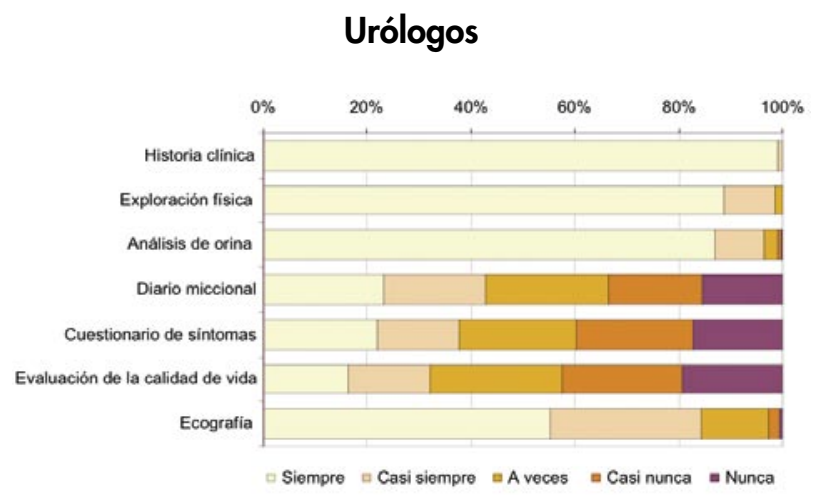

\section{RESULTADOS}

En el estudio participaron un total de 696 médicos de AP y 748 urólogos.

\section{Protocolos de actuación utilizados en urología}

Un 64,8\% de los urólogos afirmaron seguir algún protocolo estandarizado para el diagnóstico de pacientes con SVH. Casi en su totalidad lo hacían de acuerdo con las pautas establecidas por su hospital o centro de salud. Un 35\% del total de urólogos afirmaron utilizar sólo el protocolo de la AEU, un $14 \%$ sólo el protocolo de la EAU, un $9 \%$ protocolos propios, un $4 \%$ sólo el protocolo de la $\mathrm{ICl}$ y el $3 \%$ restante utilizaban más de un protocolo estandarizado.

\section{Perfil de pacientes con síntomas urinarios derivados desde AP a urología}

Un $58,4 \%$ de los médicos de AP afirmaron no derivar a los pacientes con síntomas urinarios, un $26,3 \%$ dijeron hacerlo únicamente en casos específicos, un $12 \%$ lo hacía casi siempre y un 3,3\% siempre. En cuanto a los casos específicos que motivaban la derivación del paciente al especialista según el médico de AP destacaron el diagnóstico difícil o poco claro $(91 \%)$, el fracaso terapéutico $(90,1 \%)$, la detección de anomalías en exploración física $(83,5 \%)$, los antecedentes quirúrgicos $(74,2 \%)$ y la hematuria $(66,6 \%)$, entre otras causas.

Al evaluar el perfil del paciente que llega a la consulta del urólogo, el $77,8 \%$ de los urólogos afirmaron que los pacientes llegaban sin ningún diagnóstico o estudio previo, el 35,3\% afirmaron que los pacientes llegaban con diagnóstico poco claro, el

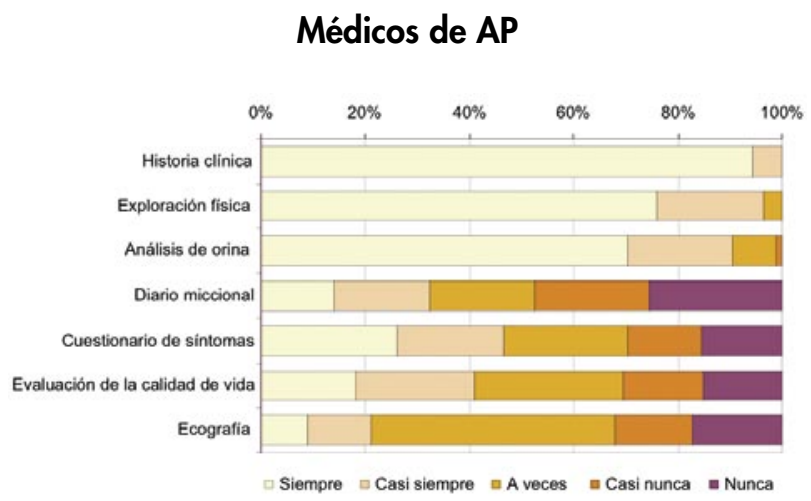

FIGURA 1. Frecuencia de utilización de pruebas y procedimientos para el diagnóstico de SVH según la especialidad médica. 
$25,3 \%$ con fracaso terapéutico, el $12,4 \%$ con prolapso y el $7,4 \%$ con antecedentes quirúrgicos. Otros motivos de derivación declarados presentaban una frecuencia baja (inferior al 5\% de los pacientes).

\section{Técnicas y pruebas diagnósticas utilizadas}

La Figura 1 muestra una descripción de los procedimientos y pruebas utilizadas para el diagnóstico de SVH. En ambas especialidades la historia clínica, la exploración física y el análisis de orina son procedimientos que forman parte de la práctica diagnóstica habitual. En el caso de los urólogos cabe añadir a dichos procedimientos la ecografía, que se realizaba siempre o casi siempre en el caso del $84,4 \%$ de los investigadores. Los procedimientos menos utilizados en ambas especialidades fueron las medidas basadas en el propio paciente o en la información reportada por el paciente como el diario miccional y los cuestionarios de síntomas o de evaluación de la calidad de vida.

Cabe destacar que el $46,7 \%$ de los médicos de AP y el $37,8 \%$ de los urólogos dijeron utilizar "siempre" o "casi siempre" una escala de síntomas siendo las diferencias observadas estadísticamente significativas $(p<0,01)$. Del mismo modo, asumiendo que los facultativos que usan el diario miccional son aquellos que dijeron usarlo "siempre" o "casi siempre", los resultados obtenidos reflejan una frecuencia estadísticamente superior en urólogos que en médicos de atención primaria en cuanto al uso del diario miccional para evaluar el diagnóstico y gravedad del $\mathrm{SVH}$ (el $42,8 \%$ frente al $32,2 \%$ respectivamente $(p<0,01))$. En este sentido, la Tabla I muestra una descripción de las preguntas incluidas en los diarios miccionales utilizados por los investigadores incluidos en el estudio. El tipo de información recogida y el tiempo de recogida de dicha información es prácticamente igual entre ambas especialidades. Entre los cuestionarios de calidad de vida más utilizados entre los facultativos, cabe destacar el King's Health Questionnaire, el EQ-5D y el ICIQ.

En el caso de los urólogos se recogió también información sobre la realización de estudio urodinámico y sobre otras pruebas y técnicas de imagen utilizadas (Tabla II). El 69,3\% de los urólogos afirmaron realizar, en condiciones de práctica

TABLA I. DESCRIPCIÓN DE LAS PREGUNTAS INCLUIDAS EN EL DIARIO MICCIONAL UTILIZADO EN CADA UNA DE LAS ESPECIALIDADES EN ESTUDIO Y TIEMPO DE RECOGIDA DE LA INFORMACIÓN.

\begin{tabular}{|c|c|c|c|c|}
\hline \multirow{2}{*}{} & \multicolumn{2}{|c|}{ Médico de AP } & \multicolumn{2}{c|}{ Urólogos } \\
\cline { 2 - 5 } & $\mathbf{n}$ & $\%$ & $\mathbf{n}$ & $\%$ \\
\hline Preguntas incluidas en el diario: & & & & \\
\hline Frecuencia de micciones & 275 & 94,2 & 352 & 89,6 \\
\hline Horario de micciones & 190 & 65,1 & 270 & 68,7 \\
\hline Volumen de micciones & 179 & 61,3 & 250 & 63,6 \\
\hline Episodios de urgencia & 241 & 82,5 & 321 & 81,7 \\
\hline Episodios de incontinencia & 247 & 84,6 & 316 & 80,4 \\
\hline Material absorbente utilizado & 141 & 48,3 & 268 & 68,2 \\
\hline Ingesta de líquidos & 194 & 66,4 & 201 & 51,1 \\
\hline Tiempo de recogida de la información: & & & & \\
\hline 1 día & 14 & 4,7 & 31 & 7,6 \\
\hline 2 días & 21 & 7,1 & 30 & 7,4 \\
\hline 3 días & 55 & 18,6 & 105 & 25,9 \\
\hline 4 días & 30 & 10,1 & 23 & 5,7 \\
\hline 5 días & 42 & 14,2 & 54 & 13,3 \\
\hline 6 días & 134 & 45,3 & 163 & 40,1 \\
\hline
\end{tabular}


clínica habitual, un estudio urodinámico en función de las características de los pacientes (síntomas de incontinencia, fracaso terapéutico previo, etc...), mientras que el $28,2 \%$ lo realizaba a la totalidad de los pacientes. Además del estudio urodinámico las pruebas más utilizadas en el diagnóstico del SVH eran la ecografía (90,6\% de los urólogos), la flujometría o estudio de flujo-presión $(85,8 \%)$, la cistomanometría convencional $(67,3 \%)$ y la cistouretrografía $(54,4 \%)$. El resto de pruebas la realizaban menos de un $50 \%$ de los urólogos del estudio.

\section{Actualización de la información sobre patologías urinarias}

Las fuentes de información que con más frecuencia dijeron emplear los médicos de AP para actualizar sus conocimientos sobre patología relacionada con síntomas urinarios fueron la literatura científica y la información proporcionada por las compañías farmacéuticas $185,6 \%$ y $85,5 \%$, respectivamente). En menor porcentaje se observaron los cursos de formación $(40,5 \%)$ o las reuniones, congresos y simposios $(39,1 \%)$.

\section{DISCUSIÓN}

El presente estudio ha permitido obtener información sobre el diagnóstico y manejo clínico de los pacientes que acuden a consulta de AP o urología con síntomas urinarios sugestivos de $\mathrm{SVH}$. Algo más de un tercio de los urólogos participantes en el estudio afirmaron no seguir ningún protocolo estandarizado para el diagnóstico y manejo del SVH. Cuando sí se emplean, de entre los protocolos o guías que destacaron los urólogos, el más utilizado era el del Grupo de Trabajo de Salud Vesical de la AEU (17).

Entre los médicos de AP que afirmaron derivar sólo en algunas ocasiones, la gran mayoría dije-

TABLA II. REALIZACIÓN DE ESTUDIO URODINÁMICO Y OTRAS PRUEBAS Y TÉCNICAS DE IMAGEN UTIIIZADAS POR LOS URÓLOGOS EN EL DIAGNÓSTICO DE SVH.

\begin{tabular}{|l|l|c|c|}
\hline \multirow{3}{*}{$\begin{array}{l}\text { REALIZACIÓN DE } \\
\text { ESTUDIO URODINÁMICO }\end{array}$} & $\mathbf{n}$ & $\%$ \\
\cline { 2 - 4 } & Siempre & 209 & 28,2 \\
\cline { 2 - 4 } & En ocasiones: & 514 & 69,3 \\
\cline { 2 - 4 } & Motivo & & \\
\cline { 2 - 4 } & Síntomas de incontinencia & 218 & 30,2 \\
\cline { 2 - 4 } & Fracaso terapéutico previo & 89 & 12,3 \\
\cline { 2 - 4 } & Difícil diagnóstico & 39 & 5,4 \\
\cline { 2 - 4 } & Posible relación con intervención quirúrgica & 34 & 4,7 \\
\cline { 2 - 4 } & Nunca & 19 & 2,5 \\
\hline Cistomanometría convencional & 496 & 67,3 \\
\hline Flujometría o estudios de flujo-presión & 635 & 85,8 \\
\hline Técnicas de videourodinamia & 96 & 13,0 \\
\hline Electromiografía & 221 & 30,2 \\
\hline Técnicas de imagen: & & \\
\hline Ecografía & 678 & 90,6 \\
\hline Cistocopia & 368 & 49,2 \\
\hline Citouretrografía & 407 & 54,4 \\
\hline Resonancia magnética nuclear & 58 & 7,8 \\
\hline Videocistouretrosonografía (VCUS) & 28 & 3,7 \\
\hline Cistouretroscopia & 269 & 36,0 \\
\hline Cistografía & 270 & 36,1 \\
\hline
\end{tabular}


ron hacerlo en caso de o bien diagnóstico poco claro o bien fracaso terapéutico.

En AP el diagnóstico de SVH se basa principalmente en la historia clínica, la exploración física y el análisis de orina. Según las guías de diagnóstico y manejo del SVH el diagnóstico se debería centrar en una exploración general del paciente pero también en el diario miccional, en una escala de síntomas y en un cuestionario de calidad de vida (17-19). La exploración física del paciente debería incluir una exploración abdominal, una exploración rectal, un análisis de orina, un cultivo de orina y la determinación del volumen residual. Según los datos obtenidos en el presente estudio más de un $95 \%$ de los médicos de AP declararon realizar historia clínica y exploración física siempre o casi siempre. Las técnicas diagnósticas menos utilizadas en condiciones de práctica clínica habitual y en las que se observan más desviaciones respecto las recomendaciones es el uso de diario miccional, escala de síntomas y cuestionario de calidad de vida. Prueba de ello es que sólo entre un 30 y un $45 \%$ aproximadamente de los médicos utilizan cada una de estas técnicas.

En la consulta especializada de urología, la práctica totalidad de los urólogos afirmaron que la urodinamia formaba parte del proceso diagnóstico de los pacientes, ya fuera en la totalidad de los pacientes que acudían a consulta por síntomas urinarios sugestivos de $\mathrm{SVH}$ o en función de las características de los mismos. Según datos del presente estudio, en la consulta especializada en urología sólo dos tercios de los médicos incluye la realización de la cistometría a sus pacientes para confirmar el diagnóstico, de lo que se obtiene que, a pesar de las recomendaciones actuales, aún existe un porcentaje considerable de urólogos que no confirma el diagnóstico urodinámico mediante cistometría.

Los principales motivos de derivación reportados por ambas especialidades son bastante consistentes, manteniendo en ambos casos el mismo orden de importancia de los motivos. Sin embargo, existe una mayor divergencia en el porcentaje de pacientes que son derivados de AP a consulta especializada sin ningún estudio o diagnóstico previo según la especialidad que reporta dicha información.

El presente estudio nos aporta información relevante para conocer el proceso diagnóstico y posterior manejo de los pacientes con $\mathrm{SVH}$, aunque como toda investigación presenta algunas limitaciones que cabe destacar. La primera limitación del estudio deriva de la inclusión de urología como única consulta médica especializada, sin tener en cuenta otras especialidades que tratan habitualmente dicha condición. Una segunda limitación, es que la reali- zación de una encuesta voluntaria puede generar un sesgo de respuesta, dado que probablemente la respuesta sea superior entre los investigadores más sensibilizados o con más conocimientos sobre el problema de estudio. Esta limitación, inherente a la realización de una encuesta, se ve reducida en el presente estudio por el tamaño muestral, dado que se trata de una muestra lo suficientemente grande para que sea representativa de la población de médicos de cada una de las especialidades, y por el elevado porcentaje de participación que se ha obtenido. La tercera y última limitación a destacar deriva del hecho de tratarse de una encuesta dirigida a médicos en lugar de una recogida de datos a nivel individual de los pacientes. Al tratarse de una encuesta a médicos existe el riesgo de recoger el manejo que estos consideran adecuado pero que puede presentar alguna pequeña desviación con el manejo realizado en condiciones de práctica clínica habitual. Sin embargo, la recogida de información a nivel de paciente habría limitado, por aspectos de factibilidad, el número de investigadores participantes, reduciendo de esta forma la representatividad de la muestra.

\section{CONCLUSIONES}

El SVH constituye un problema clínico importante, tanto por su alta prevalencia como por su gran impacto en la Calidad de Vida de los pacientes.

Es fundamental, establecer estrategias de detección y manejo de los síntomas en la práctica clínica asistencial.

\section{BIBLIOGRAFÍA y LECTURAS RECOMENDADAS (*lectura de interés $y^{* *}$ lectura fundamental)}

**1. ABRAMS, P.; CARDOZO, L.; FALL, M. y cols.: "The standardisation of terminology of lower urinary tract function: report for the standardisation sub-committee of the international continence society". Neurourol. Urodyn., 21: 167, 2002.

2. HASHIM, H.; ABRAMS, P.: "Drug treatment of overactive bladder: efficacy, cost and quality-oflife considerations". Drugs, 64: 1643, 2004.

**3. MILSOM, I.; STEWART, W.; THUROFF, J.: "The prevalence of overactive bladder". Am. J. Manag. Care, 6: 565, 2000.

*4. STEWART, W.F.; VAN ROOYEN, J.B.; CUNDIFF, G.W. y cols.: "Prevalence and burden of overactive bladder in the United States". World J. Urol., 20: $327,2003$. 
*5. CASTRO, D.; ESPUÑA, M.; PRIETO, M. y cols.: "Prevalencia de Vejiga Hiperactiva en España: Estudio poblacional". Arch. Esp. Urol., 58: $131,2005$.

6. SÁNCHEZ, R.; RUPÉREZ, O.; DELGADO, M.A. y cols.: "Prevalencia de la incontinencia urinaria en la población mayor de 60 años atendida en atención primaria". Aten. Primaria, 24: 421, 1999.

7. BRETONES, J.J.; PINO, M.; GARCÍA, M.A. y cols.: "Incontinencia urinaria en mujeres en edad adulta: estudio descriptivo en una población rural". Aten. Primaria, 20: 45, 1997.

8. DAMIÁN, J.; MARTÍN-MORENO, J.M.; LOBO, F. y cols.: "Prevalence of urinary incontinence among Spanish older people living at home". Eur. Urol., 34: 333, 1998.

9. GAVIRA, F.J.; PÉREZ DEL MOLINO, J.; VALDERRAMA, E. y cols.: "Comunicación, diagnóstico y tratamiento de la incontinencia urinaria en los ancianos de una zona básica de salud". Atención Primaria, 28: 97, 2001.

*10. ESPUÑA, M.: "Incontinencia de orina en la mujer". Med. Clin., 120: 464, 2003.

11. SERELS, S.: "The wet patient: understanding patients with overactive bladder and incontinence". Curr. Med. Res. Opin., 20: 791, 2004.

12. HERZOG, A.R.; DIOKNO, A.V.; BROWN, M.B. y cols.: "Two-years incidence, remission and change patterns of urinary incontinence in noninstitutionalized older adults". J. Gerontol., 45: 67, 1990.
13. JUARRANZ, M.; TERRÓN, R.; ROCA, M. y cols.: "Tratamiento de la incontinencia urinaria". Aten. Primaria., 30: 323, 2002.

14. AMUZU, B.J.: "Nonsurgical therapies for urinary incontinence". Clinical Obstetrics and Gynecology, 41: 702, 1998.

15. NIETO BLANCO, E.; CAMACHO PÉREZ, J.; DAVILA ALVAREZ, V. y cols.: "Epidemiology and impact of urinary incontinence in women between 40 and 65 in a health area of Madrid". Aten. Primaria, 32: 410, 2003.

*16. BATISTA, J.E.; GRANDA, M.; RODRÍGUEZ, M.: "Incontinencia urinaria: pistas para un mejor enfoque". SEMERGEN, 31: 471, 2005.

**17. CASTRO DÍAZ, J.M.; JIMÉNEZ CIDRE, M.; MARTÍNEZ AGULLÓ, E. y cols.: "Consejo de Salud Vesical. Pautas de Actuación en la incontinencia Urinaria del Consejo de Salud Vesical". Edita Asociación Española de Urología, 1999.

18 THÜROFF, J.W.; HAMPEL, C.H.; HOHENFELLNER, M. y cols.: "Guidelines on incontinence". European Asociation of Urology. (EAU). 2001.

19. ABRAMS, P.; CARDOZO, L.; KHOURY, S. y cols.: "Recomendations of the international scientific commitee. Evaluation and treatment of urinari incontinence, pelvic organ relapse and faecal incontinence". $2^{\text {nd }}$ International Consultation on Incontinente, Paris, July 1-3, 2001. $2^{\text {nd }}$ Edition 2002. 